Itinéraires Itinéraires

Littérature, textes, cultures

\title{
Les Lettres françaises en 1955
}

\section{Luc Vigier}

\section{OpenEdition}

\section{Journals}

Édition électronique

URL : http://journals.openedition.org/itineraires/1388

DOI : $10.4000 /$ itineraires. 1388

ISSN : 2427-920X

Éditeur

Pléiade

Édition imprimée

Date de publication : 1 décembre 2011

Pagination : 87-104

ISBN : 978-2-296-55744-4

ISSN : $2100-1340$

Référence électronique

Luc Vigier, «Les Lettres françaises en 1955 », Itinéraires [En ligne], 2011-4 | 2011, mis en ligne le 01 décembre 2011, consulté le 01 mai 2019. URL : http://journals.openedition.org/itineraires/1388 ; DOI : 10.4000/itineraires.1388

\section{(c) ())}

Itinéraires est mis à disposition selon les termes de la licence Creative Commons Attribution - Pas d'Utilisation Commerciale - Pas de Modification 4.0 International. 


\title{
Les Lettres françaises en 1955
}

\begin{abstract}
« Les Lettres françaises en $1955 »$ proposes a study of the concerned voices in one of the most influential communist weekly journals in the post-war years. It will explore in particular the overlapping between ideology and combination of arts in a journal bearing excessive illustrations, that can be read as the cyclic system of an utopia and, at the same time, as the unfinished novel of an imaginary museum.
\end{abstract}

Keywords : Lettres françaises, communist culture, Louis Aragon, illustrations, museum without walls

Mots clés : Lettres françaises, culture communiste, Louis Aragon, illustrations, musée imaginaire

On ne relit plus guère aujourd'hui Les Lettres françaises refermées en 1972 sur un déprimant article d'Aragon, chant du cygne désespéré d'un homme blessé et épuisé 1 . Seuls les anciens militants, les chercheurs et quelques collectionneurs passionnés ouvrent encore les pages jaunies de ce qui fut l'une des tribunes les plus richement dotées de la culture communiste, au sens large, durant près de trente années. Il s'agit pourtant d'un édifice colossal qui mérite, avec tout le recul et les précautions oratoires qu'on voudra, une certaine forme de curiosité active. L'étendue du champ couvert par l'équipe éditoriale des Lettres françaises, dès la période de clandestinité ${ }^{2}$, les collaborateurs et dirigeants prestigieux, la richesse des contenus et des illustrations en font un objet éditorial exceptionnel qui devrait intéresser de

1. Le titre a depuis été réactivé par Jean Ristat et la reprise de l'esprit «Lettres françaises » s'est faite en plusieurs étapes : mensuelle de 1990 à 1993, sa publication s'interrompt pour reprendre en mars 2004 sous forme de cahier mensuel inséré dans L'Humanité. L'hebdomadaire est également accessible au format numérique à l'adresse http://www. les-lettres-francaises.fr [octobre 2011].

2. L'intégralité des Lettres françaises parues sous l'Occupation peut être lue dans l'édition en fac-similé de Georges Aillaud et François Eychart, Les Lettres françaises et Les Étoiles dans la clandestinité, 1942-1944, Paris, Le Cherche Midi, coll. « Documents », 2008. 
nombreux domaines de recherche : histoire de la littérature, histoire de la critique, histoire de l'art, histoire du cinéma et de ses techniques, dramaturgie et histoire du théâtre, architecture, sculpture, musique classique et populaire, histoire des sciences, mode, arts ménagers, sociologie, anthropologie, histoire de l'art et théorie du politique en art, critique marxiste, analyse du discours idéologique, histoire de la presse et des revues d'art, par exemple.

Qu'on soit devenu prudent ou réticent à l'égard d'une lourde imprégnation idéologique de ce journal culturel central dans le paysage communiste des années d'après-guerre comme à l'égard des limites assignées aux références artistiques ou scientifiques n'interdit pas de constater la puissance, encore aujourd'hui, de cette $e u v r e$ ramifiée, aussi surprenante dans ses prises de position violentes, conservatrices et peu nuancées sur les arts que dans sa capacité à constituer une vaste et généreuse fantasmagorie esthétique au service d'une culture populaire. Ce paradoxe permanent peut être ressenti comme manifestation d'une dualité morale radicale et irréductible : Les Lettres françaises exprimeraient à la fois le respect d'une ligne éditoriale très nette, rigoureuse et ferme empruntant volontiers les sentiers étroits d'une phraséologie marxiste redondante et la passion plus libre d'une équipe pour la création hebdomadaire d'une pédagogie généreuse dans les domaines des arts et des lettres.

Afin d'étudier ce mécanisme duel, ce paradoxe permanent de la compétence, de l'invention et du zèle idéologique, cette dialectique souvent de l'un et de l'autre dans la plupart des textes, on a choisi d'observer Les Lettres françaises sur une très courte période, l'année 1955, qui se situe à une certaine distance de l'affaire du portrait de Staline (mars 1953) et un an avant les révélations faites par Krouchtchev (février 1956), les événements de Hongrie (insurrections d'octobre 1956) et la publication par Aragon du Roman inachevé (novembre 1956) dont le titre n'est pas sans rapport avec ce roman cyclique des Lettres françaises qui, replaçant continûment son ouvrage sur le chantier, tisse avec la précision de l'obsession les motifs récurrents d'une utopie. Ces cinquante-deux numéros constituent une entité suffisamment cohérente pour servir ici d'assise à l'analyse, de même qu'on retrouverait, par hypothèse fractale, l'ensemble du discours annuel des Lettres françaises à l'échelle d'un seul numéro de douze feuilles et vingtquatre pages, chaque numéro s'efforçant vers la représentation du projet global. À l'image de ces lecteurs qui, interrogés par le journal sur leur exposition idéale, réclamaient qu'on leur signale les grandes constantes de l'histoire des arts, nous chercherons donc, dans cet ensemble de six cents pages, à la fois ce qui crée cette symphonie lourde et prévisible traversée par des voix singulières et une politique de l'image plus libre ou plus libératrice que la simple lecture des textes les plus datés ou les plus refermés sur leurs certitudes ne pourrait le laisser penser.

Les Lettres françaises disent, dans chaque numéro, à la manière d'un chœur musical, la force d'une équipe et d'une conscience collective où 
chaque membre travaillerait dans la même direction, même si les tonalités varient, de même que la subtilité des approches. Sur le plan de la tonalité et de la nature des voix, le parti pris est celui d'une vulgarisation, d'une pédagogie raisonnée, qui passent par une rhétorique de proximité : suivant le principe très fréquemment formulé à la Libération, notamment par Aragon, l'intellectuel, s'adressant aux masses - «ne cherche pas à donner le change ${ }^{3}$ » -, il sait qui il est, d'où il parle et assume cette différence radicale tout en adaptant sa parole à l'assemblée commune. À côté des articles consacrés à l'actualité politique et culturelle, à l'occasion d'un congrès d'écrivains dans telle ville du bloc de l'Est, ou bien lors de tel congrès pour la paix, qui sont d'une austérité manifeste, le ton des articles et notes consacrés à l'art ou à des questions d'esthétique est volontiers personnel, engagé, subjectif, les lourdeurs presque volontaires, jusqu'à ce qu'on comprenne que cette subjectivité se trouve assez systématiquement habitée, d'une voix à l'autre, par les mêmes inflexions idéologiques et morales, fustigeant l'individualisme, l'hermétisme, la recherche prétentieuse de l'abstraction, l'élitisme des expositions, selon une ligne ouvriériste soutenue par le fameux Lecœur qui avait valu tant de déboires à Aragon lors de l'affaire du portrait. Du même coup, la subtilité que l'on cherchait dans les affaires esthétiques ou culturelles semble se recourber, au prix parfois d'un étouffement de la pensée, vers l'austérité des articles plus spécifiquement politiques. Tout ainsi, sur les vastes pages des Lettres françaises, communique, se contamine et fait entendre une polyphonie cohérente en ménageant une forme de diversité dans la nature des approches. Cette familiarité de la voix, volontiers autobiographique, que l'on entend chez George Besson, Pierre Descargues, mais aussi chez Cocteau et Pierre Daix, participe d'une forme de colloque continu, familier, où les articles se répondent et s'entrecroisent comme s'ils émanaient d'une même instance et d'un même noyau et pour cette raison ne peut être ressentie comme de la légèreté : mêlée à ce ton de conversation érudite et amicale, qui doit plus à un lissage rédactionnel qu'à une parfaite entente des collaborateurs (les tensions sont parfois explicites), apparaît en outre une voix d'autorité qui, pour les questions esthétiques et littéraires, ne laisse rien passer. Les articles de Pierre Daix notamment sont sur ce point assez intimidants. En contrepoint des exercices de style élégants et précis qu'on peut lire dans Le Monde sous la plume d'Émile Henriot par exemple (qui sera la cible de Wurmser à la fin de l'année 1955), les textes de Pierre Daix semblent gravés dans l'airain de convictions indéfectibles et inébranlables. C'est d'autant plus difficile à accepter pour le lecteur d'aujourd'hui (et sans doute pour les lecteurs réticents de l'époque) que l'acuité du regard d'un chroniqueur de cette envergure est tout à fait sensible, par ailleurs, et parfois simultanément.

Et puis l'on découvre les articles d'Aragon qui, en termes de voix, sont situés à l'évidence sur une autre portée musicale. Lorsque le directeur des

3. Louis Aragon, L'Homme communiste, Paris, Gallimard, 1946, p. 9. 
Lettres françaises intervient, et c'est l'intérêt de considérer ce phénomène à l'intérieur du dispositif typographique de l'hebdomadaire, c'est pour de gigantesques papiers, en l'occurrence consacrés aux « Littératures soviétiques », sous la forme d'une pré-publication intitulée « Petit essai sur le $2^{\mathrm{e}}$ congrès des écrivains soviétiques » et divisé en une dizaine de chapitres de dimension respectable : trois colonnes en Une, généralement suivies de huit colonnes en page trois, quatre ou cinq, ce qui correspond à un essai de dix pages. La taille et la dimension de l'article, consacré à tel aspect de la littérature soviétique, s'expliquent en partie par la volonté d'Aragon de donner le maximum d'informations sur des auteurs méconnus ou méprisés, mais doit s'entendre aussi comme une prise de pouvoir au sein du territoire typographique de la revue. Concrètement, les articles d'Aragon rejettent les autres textes vers les marges, non seulement par la dimension, mais par la puissance de la voix. Les concessions faites par Aragon à la doxa marxiste et au réalisme socialiste, dont il parle abondamment, ne parviennent pas à masquer ou à modérer les arabesques dynamiques d'une pensée qui dépasse en qualité et en précision les tentatives de ses collaborateurs, fussent-ils du sérieux et de la dimension intellectuelle d'un Daix, d'un Sadoul ou d'un Wurmser. À l'instar de ce gigantesque article sur Delacroix (« La légende antique de la toison d'or », n 563) que publie Aragon en 1955 dans Les Lettres françaises, typographiquement et intellectuellement, c'est la voix majeure qui encadre l'ensemble des propos tenus, plus ou moins clairement, sur les missions de l'art que l'on peut détecter dans les écrivains et peintres du passé, et notamment dans les XVIII ${ }^{\mathrm{e}}$ et $\mathrm{XIX}^{\mathrm{e}}$ siècles, qui restent les siècles de référence pour les marxistes des Lettres françaises. Directeur, voix dominante et cadrante au cœur du chœur, Aragon en est aussi, à plus d'un titre, le témoin, la mémoire et l'esprit. Il est la voix des grands hommages (en 1955 comme dans les dix-sept années qui vont suivre), à la littérature soviétique alignée, certes, mais aussi aux grands auteurs français qu'il a connus. En 1955, Paul Claudel sera ainsi salué dans un tonalité toute différente des articles convenus sur le camp adverse, solennelle, libre et prenante :

Un grand poète vient de mourir. Un très grand écrivain. On sait assez tout ce qui nous séparait de lui. Il m'avait dit, en 1944, à Lyon, dans cette soirée que nous avions passée avec lui, Elsa, André Rousseaux et moi : « Je déteste tout ce que vous défendez. » Cela ne trouvait pas en moi la réponse qu'il imaginait : car je ne déteste pas tout ce qu'il aimait, et sa phrase oubliait simplement la France, qui était entre nous un lien. J'ai écrit l'an dernier que : ...J'ai souvent envié le vers de Paul Claudel... Ce n'est pas aujourd'hui que je m'en dédirai. Il était un homme de sa classe, et sur ce plan-là, il n'y avait pas de conciliation possible entre lui et nous. Mais il demeure que, par lui, s'est exprimée avec une force extraordinaire, une grandeur qui est celle de notre peuple, une voix venant de la profondeur de notre histoire, et cela fait que rien n'effacera Claudel de la littérature française. Que demain rendra justice à une œuvre encore aujourd'hui toute partagée de nos luttes. (« Mort d'un poète ", $\mathrm{n}^{\circ} 557$ ) 
La voix d'Aragon, parmi d'autres voix moins souples, contribue ainsi, par sa puissance et sa mélodie propre, à recentrer (par instants) le débat sur des questions susceptibles de dépasser les clivages et les clôtures ou tout au moins de créer des respirations, des surprises, des bifurcations inattendues du jugement dans un univers aux routes toutes tracées, ce dont semble attester son texte « Les Temps nouveaux », où il n'est pas besoin de savoir lire entre les lignes pour entendre à la fois l'alignement sur les antiennes attendues ${ }^{4}$ et la volonté d'un dégel interne sur la question du réalisme:

$[\mathrm{N}]$ ous nous refusons à considérer le réalisme socialiste comme une chose fixée, à laquelle on peut s'habituer, avec laquelle on peut composer, qui est affaire de clé à mettre dans la serrure pour l'ouvrir, comme un système dans lequel on s'inscrit, pour peu qu'on en ait saisi le mécanisme, etc. » $\left(\mathrm{n}^{\circ} 558\right)$

On ne tentera pas ici d'atténuer la rigidité des prises de position aragoniennes en 1955, mais force est de constater que la voix du maître d'œuvre des Lettres françaises bouge sans cesse autour de concepts constamment repris et réécrits, repensés à partir d'une nouvelle œuvre ou d'une nouvelle publication critique. On ne saurait avoir une image exacte des lignes idéologiques des Lettres françaises dans les années 1950 si l'on ne prend pas en compte, globalement, les états successifs d'une pensée qui travaillera, de l'intérieur, les idées les plus sclérosantes de l'esprit de parti en art.

Sur ce corpus très restreint, comme sur l'ensemble des numéros parus jusqu'au début des années 1960, le dispositif éditorial se ressent assez immédiatement comme « lieu de mémoire » où se télescopent les références historiques et culturelles, mettant en relation toutes les époques. Ce travail d'anamnèse et de commémoration des Lettres françaises se détecte dans les attaches très vives entretenues par l'équipe de rédaction avec la Résistance communiste d'une part, avec la culture artistique française d'autre part, dont les ramifications sont à la fois très étendues dans le temps et l'espace et précisément contrôlées sur le plan théorique et idéologique. Si dialogue il y a avec ce que Maurice Halbwachs nommait la « mémoire collective ${ }^{5}$ », elle se greffe tout d'abord sur le territoire mémoriel encore très proche du rôle des communistes sous l'Occupation dont elle se fait le gardien. La bannière fonctionne ainsi comme stèle commémorative et rappelle le nom du fondateur - « Jacques Decour, fusillé par les Allemands »-, indiquant à partir de février 1953, celui d'Aragon comme directeur. Cet héritage symbolique rattache le journal au souvenir des martyrs de la Résistance, au premier plan desquels les « Fusillés de Châteaubriant », tels que les

4. « Nous considérons le réalisme socialiste non pas comme mot d'ordre passager mais comme la conception enfin trouvée à partir de laquelle commence la science littéraire [...] nous le croyons la base définitive de l'art. »

5. Maurice Halbwachs, La Mémoire collective [1950], édition critique établie par Gérard Namer, Paris, Albin Michel, coll. «Bibliothèque de l'Évolution de l'Humanité », 1997. 
a évoqués Aragon et qui sont régulièrement rappelés, par incises ou par allusions, au lectorat. L'importance de la valeur testimoniale représentée par Aragon se lit par exemple, quatorze ans après le drame, dans un article très dense de Georges Sadoul sur Les Évadés de Jean-Paul Le Chanois qui conclut sur l'importance d'un sujet cinématographique de cette nature :

Et croit-on qu'il existe en France, dans les temps modernes, un plus beau sujet de tragédie que les fusillés de Chateaubriant! Dans l'inoubliable récit qu'Aragon rédigea dans la clandestinité, un scénariste trouverait, avec la condensation dramatique apportée par " la règle des trois unités ", un sujet héroïque, exaltant pour tous, montrant la diversité de notre patrie dans l'union avec des héros qui ont depuis longtemps, comme Jeanne d'Arc, leurs rues et leurs monuments partout, de J.-P. Timbaud à Guy Môquet. (Georges Sadoul, $\mathrm{n}^{\circ} 575$ )

Ceci crée un enchâssement singulier, où les cultes de l'homme de lettres et du témoin se croisent et forment, dans le territoire du souvenir et de la fidélité aux morts, une inclusion supplémentaire de mémoire vive. Dans cet article de Sadoul, on aperçoit même trois niveaux de mémoire : celui de l'auteur du journal qui fut à l'origine du film (Michel André) et qui y jouera un rôle; le film comme lieu de mémoire des prisonniers de guerre et des déportés; et l'article comme contact avec la mémoire générale de la résistance communiste et de ses sacrifices, évoquée par Sadoul à travers l'exemple du texte d'Aragon. Ainsi mêlées, les strates finissent par constituer un tissu de mémoire singulièrement dense et itératif où les formules figées, les représentations de type votif et iconique n'entravent pas, au détour d'une page, le surgissement de l'émotion contemporaine, dans la mesure où le travail de mémoire est systématiquement indexé à ce qui lui donne sens dans le présent. C'est encore sur ce point Georges Sadoul, l'un des pivots intellectuels des Lettres françaises qui en fait une synthèse théorique que l'on peut retrouver à intervalles réguliers dans les années qui vont suivre :

Le grand intérêt des Évadés est leur contemporanéité. Ce mot qu'aimait employer Poudovkine est parfaitement formé et le rigoureux Littré l'a admis sans restriction. [...] Or, la contemporanéité est une notion qui dépasse les « sujets pris sur le vif », mais s'étend à des événements anciens possédant une profonde résonance dans notre époque. Comme l'écrivait Victor Hugo dans ses Odes et ballades: Ainsi d'un peuple entier je feuilletai l'histoire / Et je sentais frémir mon luth contemporain / Chaque fois que passait un grand nom... [...] Le frémissement n'est pas propre au poète, il saisit tout le public, quand une évidente métaphore s'impose pour lui entre l'histoire et son présent. Bel-Ami a été victime de l'extraordinaire contemporanéité de son roman ${ }^{6}$. [...] Mais le détour n'est pas toujours indispensable, loin

6. Georges Sadoul fait ici allusion à l'opération de censure dont a été victime le film de Louis Daquin en 1955, ce qui déclencha, dans Les Lettres françaises, un texte de protestation collective de grands cinéastes français. 
de là. La source de la contemporanéité est dans l'événement contemporain, et le réalisateur ne doit jamais renoncer à montrer directement notre temps. $\left(\mathrm{n}^{\circ} 575\right)$

Il s'agit bien, dans cette " résonance », dans cet examen des résurgences testimoniales, d'un travail assidu de préservation des cadres sociaux de la mémoire collective dont parle Halbwachs, c'est-à-dire de la réactivation régulière, en littérature, au cinéma et dans les arts visuels en général, conçue pour être sensible dans chaque numéro, de cette mémoire particulière des groupes qui ont été marqués par un événement vécu en commun et dont la mémoire individuelle tient à la fois des souvenirs personnels et à ce que les témoins contemporains ont construit en vous et avec vous de la représentation de l'événement. Au «Témoin des Lettres », rubrique tenue chaque semaine par Pierre Abraham, répond ainsi le témoin des témoins, Aragon, mais plus souvent Pierre Daix qui, dans le numéro 574 par exemple, propose un tour d'horizon particulièrement rigoureux des livres consacrés aux camps de concentrations parus en 1955, sous le titre « Le temps des témoignages ». C'est un sujet particulièrement sensible pour Pierre Daix, non seulement parce qu'il fut lui-même déporté, témoin et qu'il écrivit sur cette expérience, mais parce que la question du témoignage porte en elle celle de sa crédibilité. C'est enfin un sujet complexe et un terrain risqué, dans la mesure où les témoignages sur les camps nazis risquent de réactiver le souvenir de dénonciations anciennes, lors du procès Kravchenko de 1949, où Les Lettres françaises furent poursuivies en diffamation et où Pierre Daix lutta pendant des semaines contre les déclarations de Kravchenko sur les camps soviétiques, au motif que ceux-ci ne pouvaient être qualifiés de camps de concentration. Cela n'empêche pas Pierre Daix d'être d'une redoutable efficacité dans la détection des faiblesses des témoignages qu'il a consultés et dont il rend compte avec une grande précision.

La mémoire des Lettres françaises s'élabore également à partir de son discours sur les arts passé au crible systématique de «l'esprit de parti » (dont on verra plus loin qu'Aragon en fait d'une étrange manière l'apologie en 1955) et d'une certaine forme de réalisme socialiste liée à l'idée du témoignage juste, que cela vienne de Pierre Descargues, de George Besson, de Pierre Daix ou d'Aragon. Il s'agit de concentrer le propos sur la part identitaire détenue par l'œuvre commentée, c'est-à-dire sa capacité à exprimer le lien profond d'un auteur et de la société au sein de laquelle elle naît. Les travaux consacrés par Les Lettres françaises aux tableaux de Bonnard en 1955 , relèvent de cette nature et l'on peut suivre la cohérence de cette méthode d'approche de l'art de toute l'équipe responsable de la rubrique «Arts » dans les propos de l'artiste et critique Francis Jourdain ( ${ }^{\circ}{ }^{574}$ ) qui, après avoir qualifié Bonnard de « matérialiste sensuel » synthétise une bonne part des enjeux soulevés par l'hebdomadaire que l'on retrouvera, presque dans les mêmes termes, sous la plume d'Aragon, dans un texte de 1959 consacré à Géricault : 
Sans rien perdre de sa valeur d'ensorceleur, ce décorateur surprenant est devenu un vrai grand peintre, un de ceux dont on peut affirmer que, s'il n'avait pas existé, l'école française ne serait pas ce qu'elle est, un peintrepeintre et aussi un fidèle témoin de son temps.

Pour témoigner de son temps, ni la volonté ni la bonne volonté ne suffisent. Si Bonnard y a réussi, ce n'est pas tout à fait intentionnellement, à la suite d'une décision spontanée ou longuement délibérée. C'est bien plutôt parce que le destin du grand artiste est - qu'il le veuille ou non et souvent médiatement - de rendre sensible le climat des jours qu'il a vécus, leur couleur et leur parfum. Ne cherchez pas, dans l'œuvre de Bonnard, l'illustration des événements qui furent des étapes importantes de notre histoire, mais il faudrait être aveugle pour n'y pas trouver la trace d'un certain état - état d'esprit, état de choses - créé par la conjoncture. Ce témoignage est involontaire, je veux dire que son authenticité est bien moins dans l'heureux choix des motifs que dans la manière de les traiter, dans la qualité même de l'art de Bonnard ${ }^{7}$.

Ce génie testimonial de l'art, capable de témoigner indirectement et involontairement (que l'on retrouve assez systématiquement dans le domaine littéraire ou un Jules Vallès, par exemple, qualifié par Jeanine Bouissounouse de "témoin indigné » pour Les Réfractaires dans le numéro 572), recoupe ce qu'Aragon nomme la « question nationale », c'est-à-dire les liens inaliénables que les artistes entretiennent avec leur nation d'appartenance, et qui les rend plus aptes que quiconque à décrire l'identité et la nature propre de leur pays. Cela relève paradoxalement d'une forme de transcendance : l'idée de nation, l'ancrage français, l'identité de l'œuvre et du pays sont des concepts qui autorisent Aragon à se dire « barrésien » en 1948 dans Les Lettres françaises, suivi en cela par un bel article de Pierre Daix sur Barrès en $1955^{\circ}$, de même que l'hommage à Claudel se fait en partie sur ce point commun d'un lien viscéral à la France. C'est du même coup tout l'hebdomadaire qui fonctionne comme gardien de l'art français, avec une insistance et une force qui ne peuvent se comprendre aujourd'hui qu'au contact de l'effroi qui s'est emparé des intellectuels français pendant toute la période de l'Occupation où un André Rousseaux, dans Les Lettres françaises clandestines, rappelait que c'est la « civilisation » française qui avait bien manqué de disparaître, ce qui se comprend si l'on se souvient de la violence des textes d'Aragon à l'égard du pillage des œuvres d'art françaises par les troupes d'occupation allemandes et dont l'auteur de L'Enseigne de Gersaint réclame le retour, sur un ton qui ne laisse aucune place au pardon et qui, bien au contraire, rappelle que le lien entre la nation et sa peinture implique que

7. Louis Aragon, "Sur Géricault », dans J'abats mon jeu [1959], Paris, Les Lettres françaises/Mercure de France, 1992, p. 105.

8. Plus exactement un commentaire critique d'un livre de Domenach sur Barrès, puisque c'est souvent indirectement que dans l'hebdomadaire, on atteint son objet, sur le modèle formulé par Wurmser de la critique de la critique. 
ces tableaux-là soient restitués au peuple français ${ }^{9}$. Au bout du compte, l'ensemble de la politique des arts dans Les Lettres françaises relèverait d'une référentialité systématique à l'idée transcendante de l'art français, dispositif bien peu matérialiste et proche, au contraire, d'une théorie des idées dont les paysages français, les portraits d'hommes et de femmes françaises, les fresques, les tapisseries, les vitraux, les sculptures d'artistes nationaux seraient les incarnations visuelles cycliques. La répétition des conditions de production de l'œuvre d'art, toujours liées selon les critiques récurrents à l'appartenance à un groupe social, à un contexte économique et politique, à un lieu, un temps et une nation finit paradoxalement par désincarner une conception de l'art dont la simple manifestation, sur le papier journal, tend à fonctionner comme un discours plus autonome et moins contrôlable. De manière plus générale, la ligne éditoriale des Lettres françaises se consolide pourtant à chaque numéro et la diversité des sujets culturels abordés aboutit à l'impression d'un grand désordre contrôlé.

L'année 1955 offre à cet égard un bon aperçu des centres d'intérêts multiples d'un hebdomadaire qui se concentre sur tout ce qui concerne les arts, les spectacles et la culture, mais aussi les sciences, les modes et les tendances ${ }^{10}$, lieux stratégiques d'une traduction concrète des principes de la lutte communiste pour l'instauration de nouvelles valeurs. La bannière de Une, qui évoluera peu dans les années 1950, inscrit cette vocation culturelle et éducative en blanc sur fond vert (la seule couleur alternative au noir et blanc à cette époque), autour du titre « Les Lettres françaises ». Dans des ellipses de lumière, on lit très clairement «Arts » (qui a remplacé le cahier " Tous les arts » dont Aragon était auparavant le directeur) et « Spectacles », masquant ainsi le volet scientifique, qui s'est imposé progressivement dans les pages intérieures, masquant aussi un déversement pléthorique de données culturelles. Classés par rubriques - « Sur les écrans de Paris », « À travers les galeries », « Revue des revues », « Théâtre », " Architecture », «Arts »-, les sujets n'en produisent pas moins une impression de variété et de richesse qui confine rapidement à la saturation de la page et des esprits, de même qu'une forme de confusion des genres et des arts s'installe au fil de la lecture, même à l'échelle d'un simple numéro de douze pages, confusion qui finit par faire sens sur une année entière.

On passe ainsi d'un article sur l'opéra de Pékin ( $\left.\mathrm{n}^{\circ} 572\right)$ à un texte très précis sur les céramiques où se trouve posée la question des frontières de l'art, et à un vaste article de George Besson sur Renoir ${ }^{11}$, dont il est beaucoup question en 1955, les arts visuels dominant à peine, selon les

9. Louis Aragon, L'Enseigne de Gersaint [1946], repris dans L'Euvre poétique, vol. 4, Paris, Livre Club Diderot, 1990, p. 585.

10. On notera, dans la version numérique actuelle des Lettres françaises, la reprise de rubriques voisines : Arts, Cinéma, Inédits, Lettres, Musique, Savoir, Société, Théâtre.

11. Respectivement Henri Crespi, "La Céramique est-elle un grand art? ", n 575 et George Besson, « Douleur et génie », ibid. 
livraisons, les articles consacrés à l'architecture urbaine, aux expositions de sculpteurs contemporains ou plus anciens (Bourdelle étant très souvent convoqué). Le mélange s'effectue aussi, avec plus ou moins de prudence, entre les arts populaires et les grandes œuvres. Les propos de Georges Boudaille, à propos du «Salon populiste » $\left(\mathrm{n}^{\circ} 573\right)$, disent à la fois la volonté de tenir compte de ce type de travaux et d'en limiter la portée, dans un numéro qui titre par ailleurs en dernière page, lieu hautement stratégique en ce qu'il est l'une des principales entrées visuelles dans le journal, sur «L'œuvre gravé de Picasso » par Charles Pérussaux :

Son seul but est de rassembler chaque année et de présenter au public des peintres qui vont chercher leur inspiration et leur sujet dans un fonds commun et populaire. La rue, le travail, l'usine, les petites joies et les grandes douleurs sont les thèmes favoris de ces exposants.

Des variations dans le mélange des arts existent d'un numéro à l'autre, mais l'année 1955 atteste d'un intérêt de plus en plus manifeste pour les productions culturelles de l'industrie cinématographique, de préférence française, et si possible située à gauche. Le début de l'année est ainsi occupé par l'affaire de la censure de Bel-Ami de Louis Daquin, plus tard il sera question du Napoléon restauré d'Abel Gance projeté sur trois écrans ou encore de French Cancan de Jean Renoir. Le cinéma américain n'est pas totalement évincé (on lit ainsi des analyses serrées de Fenêtre sur cour de Hitchcock) mais les notes le concernant restent typographiquement marginales pour une année où des chefs-d'œuvre voient le jour Outre-Atlantique, comme La Nuit du chasseur de Laugthon ou À l'est d'Éden d'Elia Kazan. La page « Sur les écrans » des Lettres françaises reste cependant d'une belle densité analytique et promeut un cinéma français indépendant qui sache résister au déferlement des productions américaines. Les notes critiques se présentent sur une demi-colonne et utilisent une signalétique que ne renierait pas aujourd'hui un hebdomadaire comme Télérama : une tête de vache plus ou moins souriante signalant le degré d'adhésion des critiques. Tout en se concentrant sur les films représentatifs de la culture française (et, dans le cas de Daquin, un film français inspiré par un roman français fustigeant les méthodes perverses d'ascension sociale d'un individualiste forcené au cœur du milieu de la presse et du journalisme), Les Lettres françaises, sous l'impulsion de Georges Sadoul, conservent un intérêt constant pour les progrès techniques de ce médium en plein essor. Accompagnant de vastes articles de fonds, on trouve également une rubrique « Radio-Télévision » indiquant les émissions recommandées par l'équipe et des comptes rendus précis sur certains reportages. On se consacre par ailleurs volontiers au théâtre, et Elsa Triolet propose par exemple de longues analyses des pièces de Bertold Brecht, à l'occasion du festival dramaturgique de Paris, où l'on donne Le Cercle de craie caucasien, ou encore pour des aperçus plus théoriques : " Ma conception du théâtre / I. Le théâtre peut-il nous restituer le monde d'aujourd'hui? » ( $\left.\mathrm{n}^{\circ} 576\right)$. Il y a de quoi se perdre dans 
les pages des Lettres françaises qui sont à la fois rigoureusement tenues et incroyablement foisonnantes. C'est pourquoi nous voudrions isoler ici deux processus cycliques majeurs qui dominent, à côté du fond de roulement des articles plus spécifiquement politiques : tout d'abord une forme d'anthologie littéraire active, qui rapproche assez nettement le dispositif de la revue du modèle du livre ou du manuel, et la constitution des Lettres françaises en musée imaginaire ensuite, intégrant l'intuition malrucienne au dispositif iconique de la revue.

À plus d'un titre, l'hebdomadaire fonctionne comme une sorte d'anthologie ou de recueil destiné à l'éducation populaire et les efforts de l'équipe consistent à tisser des liens plus ou moins cohérents entre la littérature populaire et la grande littérature, de même qu'elle tente de créer un maillage entre les grandes références des siècles passés et le « réalisme » contemporain. Les Lettres françaises fonctionnent selon des principes comparatistes avant la lettre en jouant des juxtapositions et pontages entre culture française et cultures étrangères, à l'exemple de ce que suggère ce papier de Muni, «Quand les poètes du Bengale reçoivent les poètes de la France » dans le numéro 573. Plus généralement, le comparatisme est volontiers européen et l'on publie en feuilleton aussi bien un roman sentimental à coloration sociale et féministe comme Amelia d'Henry Fielding (1751) que Nicoara fer à cheval du très stalinien romancier roumain Mihaïl Sodoveanu, considéré comme l'incarnation du réalisme socialiste roumain. Ailleurs, on célèbre en grande cérémonie les cent cinquante ans de la naissance d'Andersen ( ${ }^{\circ}$ 579), de même qu'on célèbre tous les anniversaires possibles, puisque chaque date est l'occasion pour Les Lettres françaises de rendre sensible dans le contemporain l'héritage culturel et le patrimoine tout en constituant, au cœur des pages, une forme d'atemporalité des formes, des genres et des sujets qui se croisent et se recroisent autour de quelques valeurs humaines universelles, ou bien se rencontrent autour d'une conception voisine du « réalisme ». Si l'on se situe souvent dans des univers chronologiquement assez proches (œuvres théâtrales de Brecht, toujours rattachées à la question du social-réalisme, poésie de Verhaeren), si Les Lettres rendent régulièrement hommage à des figures importantes de la première moitié du $\mathrm{XX}^{\mathrm{e}}$ siècle comme Romain Rolland, Maïakovski ou Barbusse, on voyage aussi facilement dans le temps, d'une page à l'autre, et d'un numéro à l'autre, en un mouvement perpétuel qui peut donner le vertige.

Le bicentenaire de la mort de Montesquieu fournit en 1955 le prétexte d'une convocation des grandes figures du XVIII siècle : ainsi, à côté du sentimentalisme féministe de Fielding, on trouve de larges extraits de L'Esprit des lois. Par ailleurs, dans le numéro 565 (21 au 27 avril 1955), un « reportage » sur Montesquieu est proposé par Marc Soriano qui se présente sous la forme d'un entretien et, à l'occasion de comptes rendus d'ouvrages sur Diderot, Voltaire et Fontenelle, Pierre Daix prend position pour ou contre les ouvrages universitaires publiés à l'occasion du bicentenaire. Ce lien 
dans Les Lettres françaises avec les universitaires serait à étudier de plus près : il ferait apparaître une forme de considération et de respect mais également une volonté de corriger ou de rectifier ce que cette pensée encapsulée des chercheurs de l'Université peut receler d'ignorance d'une approche marxiste des œuvres. Le XVIII ${ }^{\mathrm{e}}$ siècle continue d'influencer des articles ultérieurs, comme celui qu'Albert Dauzat consacre à l'écriture et au style du scientifique Jean Rostand ( $\mathrm{n}^{\circ}$ 574), élargissant la problématique aux écrits scientifiques des Lumières, ce qui est bel exemple de conjonction de lignes éditoriales : rendre compte des sciences, de leur valeur dans le champ idéologique, réfléchir sur le style, lier les pensées matérialistes entre elles.

Plus tôt dans l'année, comme un commentaire indirect des événements de Pologne (qui eux-mêmes auront ensuite des incidences sur les mouvements étudiants en Hongrie), la rédaction rend hommage, et Aragon en personne, à Adam Mikiewicz ${ }^{12}$, et la semaine précédente consacrait plusieurs pages à «La poésie dix ans après Desnos », présentant des poèmes inédits de Desnos et plaçant l'ensemble en relation avec la poésie contemporaine d'un Depestre interagissant avec la pensée aragonienne de la poésie, alors que Tzara rend hommage à la logique de sa destinée tragique $\left(\mathrm{n}^{\circ} 573\right)$. On pourrait peut-être apercevoir dans ce foisonnement érudit comment Les Lettres françaises se constituent justement en université, à la manière aussi de ce qui a pu se faire dans les camps de prisonniers et qu'évoque Jorge Semprun dans L'Écriture ou la vie ${ }^{13}$. Cette effervescence ne peut se départir d'un état d'esprit qui est bien celui d'une défense et illustration de la culture nationale et les explorations du patrimoine littéraire ne se font jamais sans lien avec une forme de vigilance globale se transformant souvent en acte d'accusation dirigée contre des adversaires indéterminés dont on dénonce, sous la forme d'une adresse globale, la mainmise sur la culture et sur le sens qu'ils donnent aux éléments fondamentaux de ce patrimoine.

Dans un très long article du numéro 587, André Wurmser propose en amorce de Une, sous le chapeau « Où en est notre littérature ${ }^{14}$ ? », une analyse qui reflète assez clairement le filtre à travers lequel est envisagée la littérature, dont on se surprend à penser qu'il correspond en partie à une vision très proche de celle de l'engagement et de la situation chez Sartre ${ }^{15}$, à ceci près que l'accent est mis de manière très appuyée sur la condamnation de l'individualisme de conscience, que Wurmser avait déjà dénoncé chez Paul Léautaud par le terme d'égotisme dans le numéro 562 :

12. « Je salue en lui l'éternel printemps de Pologne» (« Le Printemps polonais », n 566).

13. Jorge Semprun, L'Écriture ou la Vie, Paris, Gallimard, 1994.

14. Couvrant «Les écrivains et le sentiment national en 1955 / Robert Merle et Jules Roy devant l'Algérie / Par Pierre Daix » et le sien sous le titre « Critique de la critique ».

15. À la fois courtisé en 1955 à partir du projet de collaboration des auteurs des Lettres françaises et des Temps modernes lorsqu'il s'est agi de présenter un numéro commun de cinq cents pages sur « La Gauche », puis condamné lorsque Pierre Daix fut évincé du projet. 
Ce que la littérature a de plus précieux, c'est qu'elle nous aide à progresser dans la connaissance des hommes. Les grands réalistes (même lorsque Balzac tire parti de ses propres aventures, même lorsque Stendhal se projette dans une certaine mesure en Julien, Fabrice ou Lucien Leuwen, même lorsque Dickens insuffle sa jeunesse à David Copperfield) ont étudié l'homme dans ses rapports avec ses semblables, c'est-à-dire dans sa vie totale, réelle. Pendant le siècle qui suivit, l'exacerbation de l'invidualisme, qui va de pair avec un régime de la concurrence, poussa les écrivains à se cantonner en eux-mêmes, à s'analyser, à se chercher.

"L'homme dans sa vie totale, réelle », " dans ses rapports avec ses semblables ", voici qui correspond au mot d'ordre général de ce que les critiques des Lettres françaises considèrent comme relevant d'une littérature sociale, éloignée de la stérilité d'un Gide, érigeant la recherche du moi en principe, suivant le précept « bourgeois » de «l'homme, c'est moi » ${ }^{16}$.

Nous sommes ici au cœur de la «trahison des clercs » telle que la formulait Julien Benda en 1927 (transgression par le savant de son statut sacré de gardien rigoureux du savoir au profit d'une prise de parole dans le champ des « passions politiques »), position dont il reviendra progressivement, au profit des engagements communistes, au point que l'année 1955 des Lettres françaises lui rend régulièrement hommage, jusqu'au Prix de l'unanimité qui lui est décerné (n $\left.{ }^{0} 555\right)$. De longs articles de Pierre Daix sur Molière sont révélateurs de cet état d'esprit qui privilégie, quelle que soit l'époque, la détection dans les auteurs du passé des valeurs morales et esthétiques communistes parfois explicitement reliées aux textes fondateurs de Marx, Engels et Lénine sur l'art et la littérature dont Pierre Daix rend compte à partir de la publication de Jean Févrille, « Marx et Engels sur la littérature » (n० 555).

On notera également ce texte du numéro 564 où le même, par ailleurs très vif et précis dès qu'il s'agit de la littérature de témoignage sur les camps de concentration ou sur les ouvrages se rapportant à la déportation et la Résistance, produit un discours des plus orthodoxes, soulignant l'influence du réalisme de Scarron sur les pièces de Molière et l'évolution chez ce dernier des noms des personnages, qui quittent la sphère des noms de comédie pour s'inscrire dans le réel de Jourdain, Dandin ou Escarbagnasse. De même on trouve de longues synthèses sur Cervantès et Diderot, au moment où un encart vante les mérites d'un ouvrage de M.-D. Tsebenki, La Lutte des matérialistes français du XVIII siècle, dont on peut aussi lire le sous-titre : «Idées philosophiques et conceptions sociologiques de Diderot, Holbach, Helvetius, La Mettrie. Leur lutte contre la religion et l'église » ( $\mathrm{n}^{\circ}$ 563). À l'image de ce pontage entre la lecture du patrimoine et la convergence du matérialisme de Diderot et de Marx, l'effet de brassage et

16. André Wurmser ajoute, fustigeant la littérature de l'entre-deux-guerres : « Valéry étudie le fonctionnement de son intelligence; André Gide étale et exalte ses vices, Proust [...] scrute Proust au microscope. Hors de l'introspection, pas de salut! » 
de contiguïté est permanent, puisqu'à côté de ces articles sur le patrimoine national et parfois mondial des œuvres littéraires (ensemble constitutif en lui-même d'un vrai labyrinthe culturel où Les Lettres françaises jouent le rôle du guide éclairé), on lit un nombre considérable d'articles et de notes sur la littérature en langue russe, non seulement à travers les articles massifs d'Aragon sur les littératures soviétiques, mais aussi à travers des figures emblématiques récurrentes comme celle de Vladimir Maïakovski, dont on s'autorise fréquemment la louange, dans un travail de restauration d'une mémoire où Elsa Triolet et Aragon sont profondément engagés. Le labyrinthe étant lieu de perte de soi, et le risque d'une perte des repères étant réelle dans ce dédale de références, on ménage des mises au point esthétiques qui, dans ces années-là, chez Aragon par exemple, ne vont pas sans contorsions étranges, les critiques étant confrontés au génie propre des œuvres, qui s'adaptent parfois difficilement aux outils de l'analyse marxiste.

« Témoin des Lettres », les Lettres françaises seront donc dans ces années très marquées politiquement, produisant un dispositif d'observation, de vigilance, mais aussi d'expertise sur ce qu'il convient de soutenir en matière de littérature. La revue déploie ainsi chaque semaine un éventail considérable de repères culturels illustrés et les douze pages donnent souvent la sensation de soulever l'ensemble de la tradition littéraire française connectée à la littérature mondiale. À l'écart des avantgardes, contemptrices des aventures abstraites et détachées de l'homme, l'équipe des Lettres françaises se concentre sur une sorte de socle commun de valeurs et d'enjeux esthétiques réactivés à chaque numéro.

On peut cependant en partie opposer à ce phénomène de contrôle idéologique de la littérature, qui mêle érudition réelle et raideur analytique, les choix de l'hebdomadaire en matière d'esthétiques comparées et d'histoire vivante de l'art : jouant des aires géographiques, Les Lettres françaises proposent, certes avec le souci d'une alternance rigoureusement pensée, des approches des arts d'Extrême-Orient, des aperçus sur la peinture anglaise, allemande, flamande (Rembrandt, souvent), italienne (Giorgione, Le Titien), des synthèses sur le cinéma français (par Abel Gance, dans le $\mathrm{n}^{\circ}$ 559), sur le cinéma soviétique («Cannes 1955, Le Cinéma soviétique d'aujourd'hui » par Serge Youtkevitch, $n^{\circ}$ 567) ou sur la dimension mondiale de l'art cinématographique ( « Les films de cinquante nations » par Georges Sadoul, $\mathrm{n}^{\circ} 567$ ) qui relèvent en grande partie de l'illustration idéologique. Et là encore les anniversaires sont prétextes à de vastes rétrospectives ou aperçus synthétiques. En attestent des articles comme « Cinquante ans d'histoire de la peinture » ( $\left.\mathrm{n}^{\circ} 573\right)$, ou « Cinquante ans d'art aux ÉtatsUnis » ( $n^{\circ} 563$, où Pierre Descargues condamne chez les artistes américains « l'étude forcenée de soi-même »). Les Lettres françaises pensent 1'art en le commémorant, jouant de l'anamnèse collective, ou en structurant la fiction théorique de cette mémoire, arpentant à grande échelle l'histoire de l'art. Tissant constamment des liens avec le passé, périodisant à outrance, 
le journal rattache, accapare, récupère, étudie, travaille et fouille les siècles à travers un prisme parfaitement identifiable.

Pourtant, dans l'esprit de ce numéro 568 où le « $\mathrm{XI}^{\mathrm{e}}$ Salon de mai » se trouve présenté, l'illustration est l'occasion, sur la page, d'une cohabitation fructueuse sur le plan visuel entre Fernand Léger (un numéro entier, le 582, lui est consacré à sa mort avec un splendide article d'Aragon « Le Sourire de Léger »), Bernard Buffet, Édouard Pignon (habitués des Lettres françaises) et, en bas, un vaste article sur la peinture gothique. Une autre identité ainsi se dégage, identité sémiotique du journal lui-même qui parvient à dépasser l'orthodoxie du discours en proposant un espace typographique et iconique où les ponts, les passerelles et les connexions entre les arts de tous les pays donnent une image bien plus dynamique de la vie de l'art en France et dans le monde que ne laissent transparaître les analyses. Les réponses à l'enquête du journal au sujet de l'exposition idéale (no 580$)$ qu'imaginerait le lectorat sont particulièrement instructives sur l'état d'esprit de ce " musée pédagogique » que sont devenues Les Lettres françaises. D'une part, cette enquête confirme l'intérêt très ancien du journal pour les annonces (sous la rubrique abyssale de L'Enseigne de Gersaint, redoublée par « À travers les Galeries »), comptes rendus d'expositions et reproduction des pièces centrales de ces expositions, qui transforment les pages des Lettres françaises en véritable livre d'art ou « grand imagier» de la culture communiste d'après-guerre, mais elle se connecte à un état d'esprit formulé un peu avant par Malraux, qui est celui du « musée imaginaire ». Répondant à l'enquête proposée par l'hebdomadaire («L'exposition à faire », réponses publiées dans le ${ }^{\circ} 580$ ), Marcel Grimond formule une hypothèse, une utopie d'exposition, qui n'est pas sans rapport avec l'utopie d'un musée imaginaire actif au cœur même de la revue :

J'aimerais que cette exposition fût présentée par familles, ou, si vous voulez par parentés plastiques, afin que puissent se dégager les grandes constantes éternelles qui dirigent la plastique à travers les différences de climat, de races et d'époques. Ceci afin de nous permettre de retrouver l'unité sous les divergences de surface. Peut-être même serait-il amusant et instructif de ne point indiquer la provenance et l'époque et de mettre en exemple, telle œuvre maya au contact d'une sculpture khmer.

On rejoint tout à fait ici non seulement la réalité concrète du musée de papier que sont de toute façon les Lettres françaises et par ailleurs ce que dit Malraux des possibilités offertes par les reproductions d'œuvres d'art :

Un musée imaginaire s'est ouvert, qui va pousser à l'extrême l'incomplète confrontation imposée par les vrais musées : répondant à l'appel de ceux-ci, les arts plastiques ont inventé leur imprimerie ${ }^{17}$. [...] Le monde des

17. André Malraux, Le Musée imaginaire [1947 puis 1951 et 1965], Paris, Gallimard, coll. «Folio/Essais », 2006, p. 16. 
photographies n'est que le serviteur du monde des originaux, sans doute; pourtant, moins séduisant ou moins émouvant, beaucoup plus intellectuel, il semble révéler, au sens qu'a ce mot en photographie, l'acte créateur : faire d'abord de l'histoire de l'art, une suite de création ${ }^{18}$.

Contemporain du développement spectaculaire des moyens techniques de reproduction en noir et blanc puis en couleurs des œuvres d'art, à une époque où le musée du Louvre diffuse de grands albums luxueux de ses collections, Les Lettres françaises croisent à plusieurs niveaux la définition de l'espace abstrait du musée imaginaire tel que le définit Malraux dès 1947 :

[U]n musée connaît rarement la continuité historique de l'exposition à laquelle ont contribué vingt nations, de l'album, du Musée Imaginaire dans son ensemble; aucun musée ne nous montre à ce point une longue suite d'œuvres accéder à la vie qu'elles tiennent de leur succession, comme si quelque esprit de l'art poussait une même conquête d'enluminure en vitrail, de vitrail en fresque, de fresque en tableau ${ }^{19}$.

Enfin, Les Lettres françaises semblent faire leur la leçon de Malraux sur l'effet de suggestion comparatiste de la photographie d'œuvre d'art :

[L]a photographie en noir « rapproche » les objets qu'elle représente, pour peu qu'ils soient apparentés. Une tapisserie, une enluminure, un tableau, une sculpture et un vitrail médiévaux, objets fort différents, reproduits sur une même page, perdent leur couleur, leur matière (la sculpture, quelque chose de son volume), leur dimensions, au bénéfice d'une style commun $^{20}$.

C'est dans un esprit tout à fait malrucien (même si l'on peut envisager une influence qui irait dans l'autre sens) que l'arborescence des arts se déploie dans le temps et l'espace, relevant des paradigmes esthétiques aussi dans le monde médiéval (" Pourquoi se priver des chefs-d'œuvre du moyenâge? » par Pierre Daix, $n^{\circ} 567$ ), le monde gaulois ( $\mathrm{n}^{\circ} 557$ ), romain, celte, grec, mais aussi le monde des arts premiers d'Afrique ou de l'Inde.

C'est pourquoi on ne rendra pas compte de ce que furent Les Lettres françaises si l'on n'évoque pas l'incroyable dispositif des illustrations d'art qui habitent et hantent littéralement les pages de cet hebdomadaire avec une force et une densité continues. Certaines pages consacrées aux « Dix ans de $1^{\prime}$ Union des Arts plastiques » ( ${ }^{\circ}$ 576), aux salons des Indépendants, aux salons d'Automne, ou telle exposition rétrospective de l'œuvre de Fernand Léger à Lyon (qui disparaîtra en 1955), à « Utrillo et les siens [Modigliani, Valadon] » ( $\left.\mathrm{n}^{\circ} 578\right)$ sont, pour l'époque, d'une grande générosité iconique. Le numéro 571 par exemple, propose une page entière avec

18. Ibid., p. 161.

19. Ibid., respectivement p. 162 et p. 174 [dossier d'illustrations, p. 163 à 173].

20. Ibid., p. 96. 
douze illustrations consacrées à la " jeune sculpture » et une autre sur " Picasso au Louvre ", où se côtoient onze illustrations dont une photographie de Guernica, et une reproduction de La Guerre et la Paix, élément d'une série sur les horreurs de la guerre qui sera souvent utilisée par Les Lettres françaises où les arcs analogiques courent de 1936 à la grande peur contemporaine, toujours palpable, d'une nouvelle guerre nucléaire. Ailleurs, on rend hommage à Fra Angelico ( $\left.{ }^{\circ} 585\right)$, à Giorgione, au Caravage, à l'art gothique, à l'art rupestre, aux vitraux, à la sculpture celte et gallo-romaine, et un Claude Roy peut s'aventurer, à l'occasion de la publication d'un livre d'art, dans une synthèse « De Lascaux aux Hittites ».

C'est un véritable feu d'artifice d'illustrations, d'autant plus important aujourd'hui sur le plan de l'analyse des discours idéologiques que la richesse iconographique crée, au bout du compte, un propos qui tend à excéder les limites sclérosantes de l'habillage critique marxiste : un Léger, un Picasso, et même Miailhe et Tazlitsky disent autre chose en image que ce que tentent de plaquer sur eux des analyses attendues. Il faut considérer par ailleurs que la dimension des pages $(42 \times 60 \mathrm{~cm})$ favorise la manifestation concrète de l'œuvre comme livre d'art, mais aussi affiche, espace d'exposition, que l'on peut partager en famille, tant la surface offerte par ce grand format déborde à l'évidence les limites de la lecture individuelle. Matériellement, Les Lettres françaises constituent une vitrine des arts « réalistes » de grande dimension, que l'œil peut visiter, à l'intérieur de laquelle la mémoire et la vue peuvent circuler et se perdre un instant. Les Lettres françaises, ce serait cette étrange fantasmagorie sur basse continue idéologique, s'y soumettant parfois, y résistant souvent par la seule force de sa présence, les œuvres fussent-elles limitées par la sélection réaliste.

Claude Roy, dans le numéro 573, dit son admiration pour un petit ouvrage cinétique de Rouveyre permettant, en faisant défiler du pouce les pages, de voir un Guillaume Apollinaire en mouvement et imagine un livre qui contiendrait de la même façon les dessins gravés de Picasso : « Si on feuilletait un tel livre, monstrueux et génial, à la manière du petit cinéma de poche que nous offre Rouveyre, on se trouverait en présence du plus prodigieux dessin animé de tous les temps ». Les Lettres françaises, que nous avons eu l'occasion de décrire ailleurs comme un véritable « spectacle à la lanterne magique ${ }^{21} »$, dans la mémoire visuelle de ses lecteurs, pourraient bien être aussi ce dessin animé, cette animation mentale constituée au fil des numéros ou même à l'intérieur d'un même numéro, superposant de vastes panneaux contenant souvent dix à douze illustrations de dix centimètres carrés, marquant de son grand écran illustré les rétines, les esprits,

21. "Le papier journal, l'écran et la lanterne magique », conférence des séminaires de l'Équipe Aragon, ITEM (CNRS), 2009. À paraître en 2012 dans la Revue des Sciences humaines. 
les mémoires et dépassant, nous semble-t-il, au bout du compte, la claustration idéologique pourtant savamment organisée, en résistance indirecte de l'art. L'attention de Sadoul aux liens de la peinture et du cinéma, au cinéma d'animation, aux pellicules dessinées, rencontre heureusement ici le travail de composition effectué par Aragon, Pierre Daix et l'équipe responsable des clichages qui, dans le feuilleté de la revue, semble converger vers une cinétique critique d'un regard sur l'art en mouvement perpétuel, ce qui confère au film de Clouzot sur le geste de Picasso (première technique époustouflante) une dimension métadiscursive assez puissante, dans le numéro 583.

Surface d'observation et de résonance des enjeux idéologiques de l'après-guerre, l'art est ainsi dans Les Lettres françaises le tremplin d'une adresse singulière à une conscience collective en pleine reconstruction qui prend ses racines dans la peur d'une disparition de la France, de sa culture et de la civilisation dont elle est l'incarnation, avec un objectif obsessionnel, notamment dans les années 1950 : renvoyer le lectorat à l'image cohérente et lisible de l'homme et de la femme communistes en rattachant, dans le travail d'élaboration d'une conscience nationale communiste, les concepts aux images, les textes aux illustrations, en déployant une politique de l'image aux effets inattendus qu'il convient d'explorer, en invitant les chercheurs de nombreuses disciplines à croiser leurs regards sur un objet éditorial hors du commun. 